\title{
Nonsolvability of the asymptotic Dirichlet problem for some quasilinear elliptic PDEs on Hadamard manifolds
}

\author{
Ilkka Holopainen and Jaime B. Ripoll
}

\begin{abstract}
We show, by modifying Borbély's example, that there are 3-dimensional Cartan-Hadamard manifolds $M$, with sectional curvatures $\leq-1$, such that the asymptotic Dirichlet problem for a class of quasilinear elliptic PDEs, including the minimal graph equation, is not solvable.
\end{abstract}

\section{Introduction}

In this paper we construct a 3-dimensional Cartan-Hadamard manifold of sectional curvatures $\leq-1$ where the asymptotic Dirichlet problem is not solvable with any continuous non-constant boundary data for a large class of equations

$$
Q[u]:=\operatorname{div} \mathcal{A}\left(|\nabla u|^{2}\right) \nabla u
$$

including, in particular, the minimal graph equation

$$
\operatorname{div} \frac{\nabla u}{\sqrt{1+|\nabla u|^{2}}}=0 .
$$

Examples of such manifolds were earlier constructed for the usual Laplace equation by Ancona [4] and Borbély [8] and for the $p$-Laplace equation by Holopainen [19], whereas no counterexamples, with Sect $\leq-1$, were known for the minimal graph equation (1.2). Recall that a Cartan-Hadamard manifold $M$ is a complete, connected and simply connected Riemannian $n$-manifold, $n \geq 2$, of non-positive sectional curvature. By the Cartan-Hadamard theorem, the exponential map $\exp _{o}: T_{o} M \rightarrow M$ is a diffeomorphism for every point $o \in M$. In particular, $M$ is diffeomorphic to $\mathbb{R}^{n}$. It is well-known that $M$ can be compactified by adding a natural geometric boundary, called the sphere at infinity (or the boundary at infinity) and denoted by $M(\infty)$, so that the resulting space $\bar{M}=M \cup M(\infty)$

Mathematics Subject Classification (2010): 58J32, 53C21, 31C45.

Keywords: Minimal graph equation, Dirichlet problem, Hadamard manifold. 
equipped with the cone topology will be homeomorphic to a closed Euclidean ball; see [15]. The Dirichlet problem at infinity (or the asymptotic Dirichlet problem) on a Cartan-Hadamard manifold $M$ for the operator $Q$ is then the following: given a continuous function $h$ on $M(\infty)$ does there exist a (unique) function $u \in C(\bar{M})$ such that $Q[u]=0$ on $M$ and $u \mid M(\infty)=h$ ?

We assume that $\mathcal{A}:(0, \infty) \rightarrow[0, \infty)$ is a smooth function such that

$$
\mathcal{A}(t) \leq A_{0} t^{(p-2) / 2}
$$

for all $t>0$, with some constants $A_{0}>0$ and $p \geq 1$, and that $\mathcal{B}:=\mathcal{A}^{\prime} / \mathcal{A}$ satisfies

$$
-\frac{1}{2 t}<\mathcal{B}(t) \leq \frac{B_{0}}{t}
$$

for all $t>0$ with some constant $B_{0}>-1 / 2$. Furthermore, we assume that

$$
t \mathcal{A}\left(t^{2}\right) \rightarrow 0 \quad \text { as } t \rightarrow 0+
$$

and therefore we interprete $\mathcal{A}\left(|X|^{2}\right) X$ as a zero vector whenever $X$ is a zero vector. The equation $Q[u]=0$ is interpreted in the weak sense. More precisely, a function $u$ is a solution to the equation $Q[u]=0$ in an open set $\Omega \subset M$ if it belongs to the local Sobolev space $W_{\text {loc }}^{1, p}(\Omega)$ and

$$
\int_{\Omega}\left\langle\mathcal{A}\left(|\nabla u|^{2}\right) \nabla u, \nabla \varphi\right\rangle d m=0
$$

for every $\varphi \in C_{0}^{\infty}(\Omega)$. Such function $u$ will be called a $Q$-solution in $\Omega$. Furthermore, we say that a function $u \in W_{\mathrm{loc}}^{1, p}(\Omega)$ is a $Q$-subsolution in $\Omega$ if $Q[u] \geq 0$ weakly in $\Omega$, that is

$$
\int_{\Omega}\left\langle\mathcal{A}\left(|\nabla u|^{2}\right) \nabla u, \nabla \varphi\right\rangle d m \leq 0
$$

for every non-negative $\varphi \in C_{0}^{\infty}(U)$. Similarly, a function $v \in W_{\text {loc }}^{1, p}(U)$ is called a $Q$-supersolution in $\Omega$ if $-v$ is a $Q$-subsolution in $\Omega$. Note that $u+c$ is a $Q$-solution (respectively, $Q$-subsolution, $Q$-supersolution) for every constant $c \in \mathbb{R}$ if $u$ is a $Q$-solution (respectively, $Q$-subsolution, $Q$-supersolution). Furthermore, $u$ and $-u$ are $Q$-solutions simultaneously. It follows from the growth condition (1.3) that test functions $\varphi$ in (1.6) and (1.7) can be taken from the class $W_{0}^{1, p}(\Omega)$ if $|\nabla u| \in L^{p}(\Omega)$.

We call a relatively compact open set $\Omega \Subset M Q$-regular if for any continuous boundary data $h \in C(\partial \Omega)$ there exists a unique $u \in C(\bar{\Omega})$ which is a $Q$-solution in $\Omega$ and $u \mid \partial \Omega=h$. In addition to the growth conditions on $\mathcal{A}$, we occasionally assume that

(A) there is an exhaustion of $M$ by an increasing sequence of $Q$-regular domains $\Omega_{k}$, and that

(B) locally uniformly bounded sequences of continuous $Q$-solutions are compact in relatively compact subsets of $M$. 
We want to emphasize that in this paper we do not study which operators satisfy the assumptions (A) and (B) above because our primary motivation is a nonsolvability result for the asymptotic Dirichlet problem for the minimal graph equation (1.2) which is known to fulfil all the conditions above. Indeed,

$$
\mathcal{A}(t)=\frac{1}{\sqrt{1+t}} \quad \text { and } \quad \mathcal{B}(t)=-\frac{1}{2(1+t)}
$$

satisfy (1.5) and growth conditions (1.3) and (1.4) with $A_{0}=1$ and $B_{0}=0$, respectively. Furthermore, the condition (A) for the minimal graph equation follows from Theorem 2 in [13] where the sets $\Omega_{k}$ can be chosen as geodesic balls $B(o, k)$ centered at a fixed point $o \in M$, and the condition (B) follows from Theorem 1.1 in [29] (see also Theorem 1 in [13]). We also note that $u$ satisfies (1.2) if and only if $G:=\{(x, u(x)): x \in \Omega\}$ is a minimal hypersurface in the product space $M \times \mathbb{R}$.

The class of equations considered here include also the usual Laplace-Beltrami equation $(\mathcal{A}(t) \equiv 1$ and $\mathcal{B}(t) \equiv 0)$ and, more generally, the $p$-Laplace equation $\operatorname{div}\left(|\nabla u|^{p-2} \nabla u\right)=0,1<p<\infty$, in which case

$$
\mathcal{A}(t)=t^{(p-2) / 2} \quad \text { and } \quad \mathcal{B}(t)=\frac{p-2}{2 t},
$$

and so $A_{0}=1$ and $B_{0}=(p-2) / 2$. It is well known that the properties $(\mathrm{A})$ and $(\mathrm{B})$ above hold for the $p$-Laplace equation and that (weak) solutions of the $p$-Laplace equation have Hölder-continuous representatives, usually called $p$-harmonic functions; see [17].

The main result of this paper is the following nonsolvabity theorem whose main special case is stated separately in Corollary 1.2. We want to point out that the properties (A) and (B) are not needed in the part (a) below.

Theorem 1.1. There exists a 3-dimensional Cartan-Hadamard manifold $M$ with sectional curvatures $\leq-1$ such that

(a) for any operator $Q$, with $\mathcal{A}$ satisfying (1.3), (1.4), and (1.5), the asymptotic Dirichlet problem for the equation $Q[u]=0$ is not solvable with any continuous non-constant boundary data on $M(\infty)$,

(b) there are non-constant bounded continuous $Q$-solutions on $M$ if, in addition, $Q$ satisfies the properties (A) and (B).

Corollary 1.2. There exists a 3-dimensional Cartan-Hadamard manifold $M$ with sectional curvatures $\leq-1$ such that the asymptotic Dirichlet problem for the minimal graph equation (1.2) is not solvable with any continuous non-constant boundary data, but there are non-constant bounded continuous solution of (1.2) on $M$.

The asymptotic Dirichlet problem for the Laplace-Beltrami operator has been extensively studied during the last three decades. It was solved affirmatively by Choi [10] under assumptions that sectional curvatures satisfy Sect $\leq-a^{2}<0$ and the so-called convex conic neighborhood condition holds. The latter means 
that for any pair of points $x, y \in M(\infty), x \neq y$, there exist disjoint neighborhoods $V_{x}, V_{y} \subset \bar{M}$ in the cone topology such that $V_{x} \cap M$ is convex with a $C^{2}$ boundary. Such appropriate convex sets were constructed by Anderson [5] for manifolds of pinched sectional curvature $-b^{2} \leq$ Sect $\leq-a^{2}<0$. Independently, Sullivan [30] solved the Dirichlet problem at infinity under the same pinched curvature assumption by using probabilistic arguments. In [6], Anderson and Schoen presented a simple and direct solution to the Dirichlet problem again in the case of pinched negative curvature. Important contributions to the Dirichlet problem were given by Ancona in a series of papers [1], [2], [3], and [4]. In particular, he was able to replace the curvature lower bound by a bounded geometry assumption that each ball up to a fixed radius is $L$-bi-Lipschitz equivalent to an open set in $\mathbb{R}^{n}$ for some fixed $L \geq 1$; see [1]. On the other hand, in [4] Ancona constructed a 3dimensional Cartan-Hadamard manifold with sectional curvatures bounded from above by -1 where the asymptotic Dirichlet problem is not solvable. Another example of a (3-dimensional) Cartan-Hadamard manifold, with sectional curvatures $\leq-1$, on which the asymptotic Dirichlet problem is not solvable was constructed by Borbély [8].

The Dirichlet problem at infinity has been studied also in a more general context of $p$-harmonic and $\mathcal{A}$-harmonic functions as well as for operators $Q$. In the case of the $p$-Laplace equation the Dirichlet problem at infinity was solved in [18] on Cartan-Hadamard manifolds of pinched negative sectional curvature by modifying the direct approach of Anderson and Schoen [6]. In [20], Holopainen and Vähäkangas studied the asymptotic Dirichlet problem for the $p$-Laplace equation on a Cartan-Hadamard manifold $M$ under a curvature assumption

$$
-b(\rho(x))^{2} \leq \operatorname{Sect}_{x} \leq-a(\rho(x))^{2}
$$

outside a compact set. Here $\rho(x)$ stands for the distance between $x \in M$ and a fixed point $o \in M$ and, furthermore, $a, b:[0, \infty) \rightarrow[0, \infty), b \geq a$, are smooth functions subject to certain growth conditions; see Theorem 1.3 and Theorem 1.4 below for the two important special cases of functions $a$ and $b$.

Concerning the minimal graph equation (1.2) there has been a growing interest in entire minimal hypersurfaces in product spaces $M \times \mathbb{R}$. Indeed, in [11] Collin and Rosenberg constructed harmonic diffeomorphisms from the complex plane $\mathbb{C}$ onto the hyperbolic plane $\mathbb{H}^{2}$ disproving a conjecture of Schoen and Yau [28]. This result was extended by Gálvez and Rosenberg [16] to any Cartan-Hadamard surface $M$ with curvature bounded from above by a negative constant. The method in both papers is to construct an entire minimal surface $\Sigma=(x, u(x)) \subset \mathbb{H}^{2} \times \mathbb{R}$ ( $\Sigma \subset M \times \mathbb{R}$, resp.) of conformal type $\mathbb{C}$, and thus to construct an entire unbounded solution $u$ to the minimal graph equation. Harmonic diffeomorphisms $\mathbb{C} \rightarrow \mathbb{H}^{2}(\mathbb{C} \rightarrow M$, resp.) are then obtained by composing conformal diffeomorphisms $\mathbb{C} \rightarrow \Sigma$ with harmonic vertical projections $\Sigma \rightarrow \mathbb{H}^{2}(\Sigma \rightarrow M$, resp.). In both papers the construction of an entire unbounded solution $u$ to the minimal graph equation is based on a Jenkins-Serrin type theorem [22] on the Dirichlet problem on unbounded ideal polygons. Motivated by these unexpected results, by the desire to understand minimal hypersurfaces in product spaces $M \times \mathbb{R}$, and by 
the recent research in this field (see for example, [12], [14], [23], [24], [25], [26], [27], [29]), the authors of the current paper together with Casteras extended the results obtained in [20] for the $p$-Laplacian to the minimal graph equation under curvature assumptions (1.8). In fact, their results cover the equation (1.1), with $\mathcal{A}$ satisfying (1.3), (1.4), (1.5), and conditions (A) and (B). As special cases of their main theorem (Theorem 1.6 in [9]) we state here the following two solvability results.

Theorem 1.3 (Theorem 1.5 in [9]). Let $M$ be a Cartan-Hadamard manifold of dimension $n \geq 2$. Fix $o \in M$ and set $\rho(\cdot)=d(o, \cdot)$, where $d$ is the Riemannian distance in $M$. Assume that

$$
-\rho(x)^{2(\phi-2)-\varepsilon} \leq \operatorname{Sect}_{x}(P) \leq-\frac{\phi(\phi-1)}{\rho(x)^{2}},
$$

for some constants $\phi>1$ and $\varepsilon>0$, where $\operatorname{Sect}_{x}(P)$ is the sectional curvature of a plane $P \subset T_{x} M$ and $x$ is any point in the complement of a ball $B\left(o, R_{0}\right)$. Then the asymptotic Dirichlet problem for the minimal graph equation (1.2) is uniquely solvable for any boundary data $f \in C(M(\infty))$.

Theorem 1.4 (Corollary 1.7 in [9]). Let $M$ be a Cartan-Hadamard manifold of dimension $n \geq 2$. Fix $o \in M$ and set $\rho(\cdot)=d(o, \cdot)$, where $d$ is the Riemannian distance in $M$. Assume that

$$
-\rho(x)^{-2-\varepsilon} e^{2 k \rho(x)} \leq \operatorname{Sect}_{x}(P) \leq-k^{2}
$$

for some constants $k>0$ and $\varepsilon>0$ and for all $x \in M \backslash B\left(o, R_{0}\right)$. Then the asymptotic Dirichlet problem for the equation (1.1) is uniquely solvable for any boundary data $f \in C(M(\infty))$.

Earlier solvability results of the asymptotic Dirichlet problem for the minimal graph equation were established only under hypothesis which included the condition $\operatorname{Sect}_{x}(P) \leq c<0$ (see [16], [25]). In [25] Ripoll and Telichevesky introduced the following strict convexity condition ( $\mathrm{SC}$ condition) that applies to equations (1.1). A Cartan-Hadamard manifold $M$ satisfies the strict convexity condition if, for every $x \in M(\infty)$ and relatively open subset $W \subset M(\infty)$ containing $x$, there exists a $C^{2}$ open subset $\Omega \subset M$ such that $x \in \operatorname{Int}(\Omega(\infty)) \subset W$ and $M \backslash \Omega$ is convex. They proved that the asymptotic Dirichlet problem for (1.1) on $M$ is solvable if Sect $\leq-k^{2}<0$ and $M$ satisfies the $\mathrm{SC}$ condition; see Theorem 7 in [25]. Furthermore, they showed by modifying Anderson's and Borbély's arguments that the SC condition holds on $M$ under the curvature assumption (1.9). Thus there exist two different kinds of proofs for the result in Theorem 1.4. We remark that 2-dimensional Cartan-Hadamard manifolds $M$ with Sect $\leq-k^{2}<0$ satisfy the SC condition since any two points of $M(\infty)$ can be joined by a geodesic. Thus a sectional curvature upper bound Sect $\leq-k^{2}<0$ alone is sufficient for the solvability of the asymptotic Dirichlet problem for (1.1) for 2-dimensional CartanHadamard manifolds.

All in all, it is rather surprising that asymptotic Dirichlet problems for various equations are solvable under essentially similar curvature assumptions. Moreover, 
these solvability results have been obtained by using different kinds of proofs. Indeed, Hsu [21] solved the Dirichlet problem at infinity for the usual Laplace equation under quite similar curvature conditions than those in 1.3 and 1.4 by using probabilistic arguments. In [20] and [9] the asymptotic Dirichlet problem were solved by constructing barrier functions by direct computations. In [31] Vähäkangas considered so-called $\mathcal{A}$-harmonic equations (of type $p \in(1, \infty)$ )

$$
-\operatorname{div} \mathcal{A}(\nabla u)=0
$$

and solved the asymptotic Dirichlet problem again under similar curvature assumptions. He used PDE-methods to obtain barrier functions. Above in (1.10), $\mathcal{A}: T M \rightarrow T M$ is an operator subject to certain conditions; for instance

$$
\langle\mathcal{A}(V), V\rangle \approx|V|^{p}, \quad 1<p<\infty,
$$

and $\mathcal{A}(\lambda V)=\lambda|\lambda|^{p-2} \mathcal{A}(V)$ for all $\lambda \in \mathbb{R} \backslash\{0\}$. Note that this class of equations is different from ours in the current paper, although both include the $p$-Laplace equation. We refer to the recent paper [9] for a more detailed discussion on the asymptotic Dirichlet problem for equations of type (1.1) and (1.10).

Our paper owes much to the paper [8] by Borbély. Indeed, the construction of the manifold $M$ and the idea for the proof of the existence of non-trivial bounded continuous solutions to $Q[u]=0$ on $M$ that can not be extended continuously to $M(\infty)$ are essentially due to him. On the other hand, computations and estimates for solutions to $Q[u]=0$ in Sections 4 and 6 are more involved than those for the Laplacian in [8]. For the details in the construction of the manifold $M$ we mainly refer to [19] and to the original construction [8] by Borbély. However, for the convenience of the reader we feel obliged to repeat quite an amount of details in the construction of $M$.

\section{Main results}

Our main result, Theorem 1.1, follows from the condition (a) below since it clearly implies that no non-constant bounded continuous $Q$-solution on $M$ can have a continuous extension to $x_{0} \in M(\infty)$.

Theorem 2.1. There exists a 3-dimensional Cartan-Hadamard manifold $M$ with sectional curvatures $\leq-1$ and a point $x_{0} \in M(\infty)$ such that

(a) for any operator $Q$, with $\mathcal{A}$ satisfying (1.3), (1.4), and (1.5), for all bounded continuous $Q$-solutions $u$ on $M$, and for all (cone) neighborhoods $U$ of $x_{0}$,

$$
\inf _{M} u=\inf _{U \cap M} u, \quad \sup _{M} u=\sup _{U \cap M} u, \quad \text { and }
$$

(b) there are non-constant bounded continuous $Q$-solutions on $M$ if, in addition, $Q$ satisfies the properties (A) and (B).

The claim (b) above follows from the next result. 
Theorem 2.2. Let $M$ and $x_{0} \in M(\infty)$ be as in (2.1) and suppose that, in addition to (1.3), (1.4), and (1.5), Q satisfies also the properties (A) and (B). Then there exists a family of continuous $Q$-solutions $u_{a, c}$ on $M$, with $a \in \mathbb{R}$ and $c>0$, such that

(a) $0 \leq u_{a, c} \leq c$

(b) for all $a \in \mathbb{R}$ and $x \in M(\infty) \backslash\left\{x_{0}\right\}$,

$$
\lim _{y \rightarrow x} u_{a, c}(y)=0
$$

(c) and, for all $x \in M$,

$$
\lim _{a \rightarrow-\infty} u_{a, c}(x)=c .
$$

The proofs of Theorem 2.1 and Theorem 2.2 are based on the following theorem.

Theorem 2.3. There exists a 3-dimensional Cartan-Hadamard manifold $M$ of sectional curvatures $\leq-1$ and a point $x_{0} \in M(\infty)$ with the following properties. For all operators $Q$, with $\mathcal{A}$ satisfying (1.3), (1.4), and (1.5), there exist families of functions $\varphi_{a, c}$ and $\psi_{a, c}$ in $M$, with $a \in \mathbb{R}$ and $c \geq 0$, such that $\varphi_{a, c}$ is a continuous $Q$-subsolution on $M, \psi_{a, c}$ is a continuous $Q$-supersolution on $M$, and that

(a) $0 \leq \varphi_{a, c} \leq \psi_{a, c} \leq c$,

(b) for all $a \in \mathbb{R}$ and $x \in M(\infty) \backslash\left\{x_{0}\right\}$,

$$
\lim _{y \rightarrow x} \psi_{a, c}(y)=0 \text {, }
$$

(c) and, for all $x \in M$,

$$
\lim _{a \rightarrow-\infty} \varphi_{a, c}(x)=c .
$$

Since $0 \leq \varphi_{a, c} \leq \psi_{a, c} \leq c$, we also have

(b') for all $a \in \mathbb{R}$ and $x \in M(\infty) \backslash\left\{x_{0}\right\}$,

$$
\lim _{y \rightarrow x} \varphi_{a, c}(y)=0
$$

(c') and, for all $x \in M$,

$$
\lim _{a \rightarrow-\infty} \psi_{a, c}(x)=c .
$$

In order to deduce Theorem 2.1 and Theorem 2.2 from Theorem 2.3 we state the following important comparison principle, cf. Lemma 3.18 in [17] and Lemma 3 in [25]. We refer to Lemma 2.1 in [9] for its short proof which is based on the fact that $t \mapsto t \mathcal{A}\left(t^{2}\right)$ is strictly increasing by (1.4).

Lemma 2.4. If $u \in W^{1, p}(\Omega)$ is a $Q$-supersolution and $v \in W^{1, p}(\Omega)$ is a $Q$ subsolution such that $\varphi=\min (u-v, 0) \in W_{0}^{1, p}(\Omega)$, then $u \geq v$ a.e. in $\Omega$.

As a consequence, we obtain the uniqueness of $Q$-solutions with fixed (Sobolev) boundary data. 
Corollary 2.5. If $u \in W^{1, p}(\Omega)$ and $v \in W^{1, p}(\Omega)$ are $Q$-solutions with $u-v \in$ $W_{0}^{1, p}(\Omega)$, then $u=v$ a.e. in $\Omega$.

Proof of Theorem 2.2 assuming Theorem 2.3. Let $M, x_{0} \in M(\infty)$, and the families $\left\{\varphi_{a, c}\right\}$ and $\left\{\psi_{a, c}\right\}$ be as in Theorem 2.3. Furthermore, let $\Omega_{i} \Subset M, i \in \mathbb{N}$, be an exhaustion of $M$ by $Q$-regular domains. Note that the existence of such an exhaustion is part of our assumptions on the operator $Q$ in Theorem 2.2. For each fixed $a \in \mathbb{R}$ and $c>0$, let $u_{i} \in C(M)$ be the unique function that is a $Q$-solution in $\Omega_{i}$ with boundary values $\varphi_{a, c}$ and coincides with $\varphi_{a, c}$ in $M \backslash \Omega_{i}$. By the comparison principle (Lemma 2.4), we have $\varphi_{a, c} \leq u_{i} \leq \psi_{a, c}$ in $M$. Thus the sequence $\left(u_{i}\right)$ is uniformly bounded and hence, by the assumption (B) and a diagonal process, we obtain a subsequence of $\left(u_{i}\right)$ that converges to a function $u_{a, c}$ which is a continuous $Q$-solution in $M$, satisfies $\varphi_{a, c} \leq u_{a, c} \leq \psi_{a, c}$ in $M$, and hence conditions (a)-(c) in Theorem 2.2.

Proof of Theorem 2.1 assuming Theorem 2.3. Let $M$ and $x_{0} \in M(\infty)$ be as in Theorem 2.3. Condition (b) in Theorem 2.1 follows from Theorem 2.2. To prove (a), suppose that $h$ is a bounded continuous $Q$-solution in $M, U$ is a cone neighborhood of $x_{0}$, and let

$$
b=\inf _{M} h, \quad \text { and } \quad B=\inf _{U \cap M} h .
$$

Then $b \leq B$ and we claim that $b=B$. Write $c=B-b$ and let $\left\{\varphi_{a, c}\right\}$ and $\left\{\psi_{a, c}\right\}$, with $a \in \mathbb{R}$, be as in Theorem 2.3. Then for each $a \in \mathbb{R}$ an auxiliary continuous $Q$-subsolution

$$
f_{a}=b+\varphi_{a, c}
$$

satisfies, for all $x \in M(\infty) \backslash\left\{x_{0}\right\}$,

$$
\begin{aligned}
\liminf _{\substack{y \rightarrow x \\
y \in M}}\left(h(y)-f_{a}(y)\right) & =\liminf _{\substack{y \rightarrow x \\
y \in M}}\left(h(y)-b-\varphi_{a, c}(y)\right) \\
& \geq \liminf _{\substack{y \rightarrow x \\
y \in M}}(h(y)-b)-\lim _{\substack{y \rightarrow x \\
y \in M}} \varphi_{a, c}(y) \geq 0 .
\end{aligned}
$$

Furthermore,

$$
\begin{aligned}
\liminf _{\substack{y \rightarrow x_{0} \\
y \in M}}\left(h(y)-f_{a}(y)\right) & =\liminf _{\substack{y \rightarrow x_{0} \\
y \in M}}\left(h(y)-b-\varphi_{a, c}(y)\right) \\
& =\liminf _{\substack{y \rightarrow x_{0} \\
y \in M}}\left(\left(c-\varphi_{a, c}(y)\right)+h(y)-B\right) \geq \liminf _{\substack{y \rightarrow x_{0} \\
y \in M}}(h(y)-B) \geq 0 .
\end{aligned}
$$

Hence

$$
\liminf _{\substack{y \rightarrow x \\ y \in M}}\left(h(y)-f_{a}(y)\right) \geq 0
$$

for all $x \in M(\infty)$. It follows from the comparison principle that $h \geq f_{a}$ in $M$ for all $a \in \mathbb{R}$. To be precise, suppose on the contrary that $h(y)<f_{a}(y)-\varepsilon$ for some $y \in M$ 
and $\varepsilon>0$. Let $A$ be the $y$-component of the set $\left\{x \in M: h(x)<f_{a}(x)-\varepsilon\right\}$. Then $A$ is an open set with a compact closure $\bar{A} \subset M$ by (2.1) and continuity of $h-f_{a}$. On the other hand, $h=f_{a}-\varepsilon$ on $\partial A$, and therefore $h \geq f_{a}-\varepsilon$ in $A$ by the comparison principle leading to a contradiction. Since $\lim _{a \rightarrow-\infty} \varphi_{a, c}(x)=c=B-b$ for all $x \in M$, we obtain

$$
h(x) \geq \lim _{a \rightarrow-\infty} f_{a}(x)=B
$$

for all $x \in M$. Hence $b \geq B$, and so $b=B$.

To complete the proof, we just apply the above to the bounded continuous $Q$-solution $-h$ and obtain

$$
\sup _{M} h=-\inf _{M}(-h)=-\inf _{U \cap M}(-h)=\sup _{U \cap M} h .
$$

Remark 2.6. As is seen in the proof above, only the family $\left\{\varphi_{a, c}\right\}$ is needed in order to get the non-solvability of the asymptotic Dirichlet problem.

\section{Construction of $M$ : first step}

The construction of the Riemannian manifold $M$ is up to some minor modifications (mostly in notation) essentially due to Borbély [8]; see also [4], and [7]. For the details of the construction, we refer to [19].

We start with the standard upper half space model for the hyperbolic 3-space

$$
\mathbb{H}^{3}=\left\{\left(x^{1}, x^{2}, x^{3}\right) \in \mathbb{R}^{3}: x^{3}>0\right\}
$$

equipped with the hyperbolic metric $d s_{\mathbb{H}^{3}}^{2}$ of constant sectional curvature -1 . The sphere at infinity, $\mathbb{H}^{3}(\infty)$, can be realized as the union of the $x^{1} x^{2}$-plane and the "common endpoint $\left(x^{1}, x^{2},+\infty\right)$ " of all vertical geodesics. Let $x_{0}=(0,0,0) \in$ $\mathbb{H}^{3}(\infty)$ be a point at infinity and $L$ a unit speed geodesic terminating at $x_{0}$ $\left(L(+\infty)=x_{0}\right)$ such that $L(0)=(0,0,1)$. Thus $L$ is the positive $x^{3}$-axis. We will denote by $L$ also the image $L(\mathbb{R})$. The Fermi coordinates $(s, r, \vartheta)$ along $L$ are defined as follows. For any point $x \in \mathbb{H}^{3}$, there exists a unique point $L(s)$ on $L$ closest to $x$. This determines the $s$-coordinate uniquely. The $r$-coordinate of $x$ is the distance $r=\operatorname{dist}(x, L)=d(x, L(s))$. Finally, the third Fermi coordinate $\vartheta$ of $x \in \mathbb{H}^{3} \backslash L$ is the angle $\vartheta \in \mathbb{S}^{1}$ obtained from the polar coordinate representation $x^{1}=t \cos \vartheta, x^{2}=t \sin \vartheta$ of $x=\left(x^{1}, x^{2}, x^{3}\right)$. For $x=L(s) \in L$, the third Fermi coordinate $\vartheta$ is not defined, and we will write $x=(s, 0, *)$. On $\mathbb{H}^{3} \backslash L$, the vector fields

$$
S=\frac{\partial}{\partial s}, \quad R=\frac{\partial}{\partial r}, \quad \text { and } \quad \Theta=\frac{\partial}{\partial \vartheta}
$$

form a frame, with $\{d s, d r, d \vartheta\}$ as a coframe. Furthermore, $S, R$, and $\Theta$ are commuting as coordinate vector fields, i.e., their Lie brackets vanish:

$$
[S, R]=[S, \Theta]=[R, \Theta]=0 .
$$


From now on we abbreviate $h(r)=\cosh r$ and usually write

$$
v_{r}^{\prime}=R v, v_{s}^{\prime}=S v, v_{r s}^{\prime \prime}=S(R v), \text { etc. }
$$

for partial derivatives of a function $v$.

The (standard) hyperbolic metric of $\mathbb{H}^{3}$ in Fermi coordinates is given by

$$
d s_{\mathbb{H}^{3}}^{2}=d r^{2}+h^{2}(r) d s^{2}+\sinh ^{2} r d \vartheta^{2} .
$$

The Riemannian manifold $M$ is then obtained from $\mathbb{H}^{3}$ by modifying the metric in $\Theta$-directions as

$$
d s_{M}^{2}=d r^{2}+h^{2}(r) d s^{2}+g^{2}(s, r) d \vartheta^{2},
$$

where $g: \mathbb{R} \times\left[0,+\infty\left[\rightarrow \mathbb{R}\right.\right.$ is a $C^{\infty}$-function which is positive in the complement of $L$, that is when $r>0$,

$$
\begin{aligned}
g(s, 0) & =0, \\
g_{r}(s, 0) & :=\frac{\partial g}{\partial r}(s, 0)=1,
\end{aligned}
$$

and whose partial derivatives of even order with respect to $r$ vanish at $r=0$. Thus, with respect to the Riemannian metric $d s_{M}^{2}$, we have

$$
\langle R, S\rangle=\langle R, \Theta\rangle=\langle S, \Theta\rangle=0,\langle R, R\rangle=1,\langle\Theta, \Theta\rangle=g^{2} \text {, and }\langle S, S\rangle=h^{2} .
$$

Above and in what follows $\langle\cdot, \cdot\rangle$ refers to the Riemannian metric of $M$. Furthermore, for later purposes we record the covariant derivatives of the coordinate vector fields obtained from (3.2) by a direct computation:

$$
\begin{aligned}
& \nabla_{R} R=0, \nabla_{R} S=\nabla_{S} R=\frac{h_{r}^{\prime}}{h} S, \nabla_{R} \Theta=\nabla_{\Theta} R=\frac{g_{r}^{\prime}}{g} \Theta, \nabla_{S} S=-h h_{r}^{\prime} R, \\
& \nabla_{S} \Theta=\nabla_{\Theta} S=\frac{g_{s}^{\prime}}{g} \Theta, \text { and } \nabla_{\Theta} \Theta=-g g_{r}^{\prime} R-\frac{g g_{s}^{\prime}}{h^{2}} S .
\end{aligned}
$$

It is crucial to note that all geodesic rays of $\mathbb{H}^{3}$ starting at $L$ will remain geodesic rays also in $M$, and therefore the sphere at infinity, $M(\infty)$, of $M$ and the cone topology of $\bar{M}$ can be identified with those of $\mathbb{H}^{3}$. The Riemannian manifold $M$ will then be of sectional curvature $\leq-1$ if and only if the following four inequalities hold:

$$
\begin{aligned}
\frac{h_{r r}^{\prime \prime}}{h} & \geq 1, \\
\frac{g_{r r}^{\prime \prime}}{g} & \geq 1, \\
\frac{g_{s s}^{\prime \prime}}{g h^{2}}+\frac{g_{r}^{\prime} h_{r}^{\prime}}{g h} & \geq 1 \\
\left(-\frac{g_{r s}^{\prime \prime}}{g h}+\frac{g_{s}^{\prime} h_{r}^{\prime}}{g h^{2}}\right)^{2} & \leq\left(\frac{g_{r r}^{\prime \prime}}{g}-1\right)\left(\frac{g_{s s}^{\prime \prime}}{g h^{2}}+\frac{g_{r}^{\prime} h_{r}^{\prime}}{g h}-1\right) ;
\end{aligned}
$$

see [19]. The first condition (3.4) holds as an equality since $h(r)=\cosh r$. Thus it suffices to verify conditions (3.5) and (3.7). 


\section{The operator $Q$ for functions $\varphi_{a, c}$}

The family $\left\{\varphi_{a, c}\right\}$ in Theorem 2.3 will be constructed following the idea of Borbély in [8]. For $c=0$ these functions vanish identically, therefore we assume from now on that $c>0$. We consider a family of vector fields

$$
X^{a}=R+q_{a} S, \quad a \in \mathbb{R},
$$

on $M \backslash L$, where, for each $a \in \mathbb{R}, q_{a}: M \rightarrow \mathbb{R}$ is a $C^{\infty}$ function depending only on the $r$-coordinate of a point $(s, r, \vartheta) \in M \backslash L$ and $q_{a} \mid L=0$. ¿From now on we usually omit the parameter $a$ and abbreviate $X=X^{a}$, and write $q(r)=q_{a}(r)=$ $q_{a}(s, r, \vartheta)$. All integral curves of $X$ can be extended to $L$, and therefore we will talk about integral curves of $X$ starting at a point of $L$ even though $X$ is not defined on $X$; see [19] for details. Since $X$ does not have the $\Theta$-component, the (Fermi) $\vartheta$-coordinate remains constant along integral curves of $X$. Furthermore, integrals curves of $X$ starting at $L(s)$ are rotationally symmetric around $L$; each of them is obtained from another by a suitable rotation around $L$. Denote by $\gamma_{a, s}$ any integral curve of $X^{a}$ starting at $L(s)$. Let $S_{s}^{a}$ be the surface that is obtained by rotating any $\gamma_{a, s}$ around $L$. Note also that the relation between the (Fermi) $s$-coordinate of a point $(s, r, \vartheta) \in S_{s_{0}}^{a}$ and $s_{0}$ is given by

$$
s=s_{0}+\int_{0}^{r} q_{a}(t) d t
$$

The functions $\varphi_{a, c}$ are constructed so that the surfaces $S_{s}^{a}$ are the level sets of $\varphi_{a, c}$. Thus $\varphi_{a, c} \mid S_{s}^{a}$ has a constant value $f(s)=f^{(a, c)}(s)$ depending only on $a, c$, and $s$. It is convenient to choose

$$
f(s)=f^{(a, c)}(s)=c \max \{0, \tanh (\delta(s-a))\},
$$

where $\delta=\frac{1}{2\left(1+2 B_{0}\right)}$ and $B_{0}$ is the constant in (1.4). Hence $\varphi_{a, c} \mid M \backslash M_{a}=0$, where $M_{a}$ is the open set

$$
M_{a}=\bigcup_{s>a} S_{s}^{a} .
$$

It is worth observing that surfaces $S_{s}^{a}$ for fixed $a$ are obtained from each other by a Euclidean dilation with respect to $x_{0}$ in our upper half space model of $M$ since $q_{a}$ is independent of the $s$-coordinate. More precisely, $M_{a}=\left\{t z: t \in(0,1), z \in S_{s}^{a}\right\}$, where $t z$ stands for the (Euclidean) dilation of $z$ with respect to $x_{0}$. The functions $q=q_{a}$ will be constructed in such a way that they result in smooth functions $\varphi=\varphi_{a, c}$ in $M_{a}$. As in [8] and [19], we have

$$
\begin{aligned}
\varphi_{s}^{\prime}\left(s^{\prime}, r, \vartheta\right) & =f^{\prime}(s), \\
\nabla \varphi\left(s^{\prime}, r, \vartheta\right) & =f^{\prime}(s)\left(\cosh ^{-2} r S-q(r) R\right), \\
\left|\nabla \varphi\left(s^{\prime}, r, \vartheta\right)\right| & =f^{\prime}(s) \sqrt{\cosh ^{-2} r+q^{2}(r)}, \text { and } \\
\varphi_{s s}^{\prime \prime}\left(s^{\prime}, r, \vartheta\right) & =f^{\prime \prime}(s),
\end{aligned}
$$


where the (Fermi) coordinate $s^{\prime}$ is related to $s$ by

$$
s^{\prime}=s+\int_{0}^{r} q(t) d t
$$

Note that $\left|\nabla \varphi_{a, c}\right|>0$ in $M_{a}$. Next we will compute

$$
Q\left[\varphi_{a, c}\right]=\operatorname{div} \mathcal{A}\left(\left|\nabla \varphi_{a, c}\right|^{2}\right) \nabla \varphi_{a, c}
$$

pointwise in $M_{a}$. We start with noting that, for a $C^{2}$-function $u$ (with $|\nabla u|>0$ ),

$$
\begin{aligned}
\operatorname{div} \mathcal{A}\left(|\nabla u|^{2}\right) \nabla u & =\mathcal{A}(|\nabla u|)^{2} \Delta u+\left\langle\nabla \mathcal{A}\left(|\nabla u|^{2}\right), \nabla u\right\rangle \\
& =\mathcal{A}(|\nabla u|)^{2} \Delta u+\mathcal{A}^{\prime}\left(|\nabla u|^{2}\right)\langle\nabla\langle\nabla u, \nabla u\rangle, \nabla u\rangle \\
& =\mathcal{A}(|\nabla u|)^{2} \Delta u+2 \mathcal{A}^{\prime}\left(|\nabla u|^{2}\right) \operatorname{Hess} u(\nabla u, \nabla u) \\
& =\mathcal{A}(|\nabla u|)^{2}\left\{\Delta u+2 \mathcal{B}\left(|\nabla u|^{2}\right)|\nabla u|^{2} \operatorname{Hess} u\left(\frac{\nabla u}{|\nabla u|}, \frac{\nabla u}{|\nabla u|}\right)\right\} .
\end{aligned}
$$

In particular, we have

$$
\operatorname{div} \mathcal{A}\left(|\nabla \varphi|^{2}\right) \nabla \varphi=\mathcal{A}(|\nabla \varphi|)^{2}\left\{\Delta \varphi+2 \mathcal{B}\left(|\nabla \varphi|^{2}\right)|\nabla \varphi|^{2} \operatorname{Hess} \varphi\left(\frac{\nabla \varphi}{|\nabla \varphi|}, \frac{\nabla \varphi}{|\nabla \varphi|}\right)\right\}
$$

for functions $\varphi=\varphi_{a, c}$ in $M_{a}$.

Following Borbély, we define a $C^{\infty}$-function $\beta: M \rightarrow[0, \infty)$ (denoted by $p$ in [8]) by

$$
\beta(s, r)=\frac{g_{s}^{\prime}(s, r)}{g_{r}^{\prime}(s, r) h^{2}(r)} .
$$

Writing $Y=\nabla \varphi /|\nabla \varphi|$ and computing the Laplacian as the trace of the Hessian in the basis $\{X, \Theta, Y\}$, cf. pp. 233-234 in [8] and [19], we obtain

$$
\begin{aligned}
\operatorname{div} \mathcal{A}\left(|\nabla \varphi|^{2}\right) \nabla \varphi=\mathcal{A}(|\nabla \varphi|)^{2}\{ & \frac{\operatorname{Hess} \varphi(X, X)}{\langle X, X\rangle}+\frac{\operatorname{Hess} \varphi(\Theta, \Theta)}{\langle\Theta, \Theta\rangle} \\
& \left.+\left(1+2 \mathcal{B}\left(|\nabla \varphi|^{2}\right)|\nabla \varphi|^{2}\right) \operatorname{Hess} \varphi(Y, Y)\right\}
\end{aligned}
$$

where the Hessians are obtained from (3.3) by simple computations:

$$
\begin{aligned}
& \frac{\operatorname{Hess} \varphi(X, X)}{\langle X, X\rangle}=\frac{-\varphi_{s}^{\prime}\left(h q_{r}^{\prime}+2 h_{r}^{\prime} q+h^{2} h_{r}^{\prime} q^{3}\right)}{h\left(1+h^{2} q^{2}\right)}, \\
& \frac{\operatorname{Hess} \varphi(\Theta, \Theta)}{\langle\Theta, \Theta\rangle}=\frac{\varphi_{s}^{\prime} g_{r}^{\prime}(\beta-q)}{g},
\end{aligned}
$$

and

$$
\operatorname{Hess} \varphi(Y, Y)=\varphi_{s s}^{\prime \prime}\left(h^{-2}+q^{2}\right)-\frac{\varphi_{s}^{\prime}\left(q_{r}^{\prime} q^{2}-h_{r}^{\prime} h^{-3} q\right)}{h^{-2}+q^{2}} .
$$

Hence putting these together and simplifying we arrive at the following formula. 
Lemma 4.1. The operator $Q$ for functions $\varphi=\varphi_{a, c}$ is given in $M_{a}$ by the formula

$$
\begin{aligned}
\operatorname{div} \mathcal{A}\left(|\nabla \varphi|^{2}\right) \nabla \varphi & \\
=\frac{\mathcal{A}\left(|\nabla \varphi|^{2}\right) \varphi_{s}^{\prime}}{h}\{ & \left\{\frac{g_{r}^{\prime} h(\beta-q)}{g}-h q_{r}^{\prime}-h_{r}^{\prime} q+\frac{\varphi_{s s}^{\prime \prime}\left(1+h^{2} q^{2}\right)}{\varphi_{s}^{\prime} h}\left[1+2 \mathcal{B}\left(|\nabla \varphi|^{2}\right)|\nabla \varphi|^{2}\right]\right. \\
& \left.-2 \mathcal{B}\left(|\nabla \varphi|^{2}\right)|\nabla \varphi|^{2} \frac{h^{3} q_{r}^{\prime} q^{2}-h_{r}^{\prime} q}{1+h^{2} q^{2}}\right\} .
\end{aligned}
$$

Remark 4.2. It is worth noting already at this stage that, in order to have $Q[\varphi] \geq 0$, the first term above, i.e., the one containing $\beta-q$, should be positive and dominate the others. This requirement puts strong constraints on functions $\beta, g$, and $q$.

Remark 4.3. In order to guarantee the correct boundary behavior of the functions $\varphi_{a, c}$, i.e., condition (b') in Theorem 2.3, it is enough to construct functions $q_{a}$ so that

$$
\int_{0}^{\infty} q_{a}(t) d t=\infty
$$

for all $a \in \mathbb{R}$, and that

$$
\int_{0}^{r} q_{a}(t) d t \leq b_{r}<\infty
$$

independently of $a \in \mathbb{R}$; see Lemma 5.1 in [19].

\section{Construction of $M$ : final step}

In this section we briefly describe the construction of the function $g$ in (3.1) and hence the Riemannian metric of $M$. The function $g$ will be of the form

$$
g(s, r)=\frac{1}{2} \sinh (\sinh 2 \varrho(s, r))
$$

where $\varrho$ is a $C^{\infty}$-function, with $\varrho(s, r)=r$ for $0 \leq r \leq 3$ and $\varrho(s, r) \geq r$ for all $r \geq 0$. By (4.8), $g$ and $\varrho$ both satisfy the partial differential equation

$$
\begin{aligned}
& g_{s}^{\prime}=\beta h^{2} g_{r}^{\prime}, \quad \text { and } \\
& \varrho_{s}^{\prime}=\beta h^{2} \varrho_{r}^{\prime} .
\end{aligned}
$$

Note that $\beta$ is independent of the (Fermi) coordinate $\vartheta$ and $\beta(s, r)=0$ for $0 \leq r \leq 3$ by $(5.1)$. Since $\nabla \varrho=\varrho_{r}^{\prime}(\beta S+R)$, we have $\nabla \varrho \perp\left(\beta h^{2} R-S\right)$, and therefore $\varrho$ (and hence $g$ ) is constant along any integral curve of the vector field

$$
Z=\beta h^{2} R-S
$$


Now the idea is to construct an unbounded domain $\Omega \subset M$ of the form

$$
\Omega=\{(s, r, \vartheta) \in M: r<3\} \cup\{(s, r, \vartheta) \in M: s<-\ell(r)\}
$$

such that all integral curves of $Z$ will enter $\Omega$, and then construct $\beta$ so that it vanishes identically in $\Omega$, and finally fix the "initial condition"

$$
\varrho(s, r)=r
$$

for all $(s, r, \vartheta) \in \Omega$. Note that $(s, r, \vartheta) \in \Omega$ for all $s \leq s^{\prime}$ if $\left(s^{\prime}, r, \vartheta\right) \in \Omega$. Consequently, once an integral curve of $Z$ enters $\Omega$, it will then stay in $\Omega$ forever. The function $\ell$ that appears in (5.4) is closely related to $\beta$. Then $g$, and hence the Riemannian structure of $M$, will be completely determined by constructing the functions $\beta$ and $\ell$.

While constructing $\beta$ we have to keep in mind Remark 4.2 and (4.9). This leads to the first requirement that

$$
\int_{0}^{\infty} \beta(s, r) d r=\infty
$$

for all $s \in \mathbb{R}$. For the construction of $g$ we require that

$$
\int_{r_{0}}^{\infty} \frac{d r}{\beta(s, r) \cosh ^{2} r}=\infty
$$

for all $r_{0}>3$ and $s \in \mathbb{R}$. To obtain the curvature conditions (3.5) and (3.7) we will require that

$$
0 \leq \beta \leq \frac{1}{1000}, \quad\left|\beta_{r}^{\prime}\right| \leq \frac{1}{1000}, \quad 0 \leq \beta_{s}^{\prime} \leq \frac{1}{1000}, \quad \beta \beta_{r}^{\prime} h^{3} \leq \frac{h_{r}^{\prime}}{1000},
$$

and that $\beta h^{2}$ is a convex non-decreasing function in the variable $r$, that is

$$
\left(\beta h^{2}\right)_{r}^{\prime} \geq 0 \text { and }\left(\beta h^{2}\right)_{r r}^{\prime \prime} \geq 0
$$

The function $\beta: M \rightarrow[0, \infty)$ will be of the form

$$
\beta(s, r)=\xi(s+\ell(r)) \beta_{0}(r),
$$

with smooth functions $\xi, \ell$, and $\beta_{0}$ to be described next. The function $\xi: \mathbb{R} \rightarrow$ $[0,1]$ is smooth and non-decreasing such that $\xi|(-\infty, 0]=0, \xi|[4, \infty)=1$, and that $\xi^{\prime},\left|\xi^{\prime \prime}\right|<1 / 2$, and $\xi^{\prime \prime}+\xi>0$ on $(0,4)$. The smooth function $\beta_{0}:[0, \infty) \rightarrow$ $[0, \infty)$ is constructed such that on $\left[0, r_{1}\right]$ it is a smooth non-decreasing function that vanishes identically on $[0,3]$, takes the constant (positive) value $\beta_{0}(5)$ on $\left[5, r_{1}\right]$, and it is a positive slowly increasing function on the interval $(3,5]$ so that $(5.8)$ and $(5.9)$ hold. Here $r_{1}$ is large enough such that $\beta_{0}\left(r_{1}\right) \cosh ^{2} r_{1}=\beta_{0}(5) \cosh ^{2} r_{1}>1$. Furthermore, $\beta_{0}$ is non-increasing on $\left[r_{1}, \infty\right)$, with $\lim _{r \rightarrow \infty} \beta_{0}(r)=0$, whereas $\beta_{0} h^{2}$ is an increasing strictly convex function. Finally, $\beta_{0}$ satisfies

$$
\int_{0}^{\infty} \beta_{0}(r) d r=\infty, \quad \int_{r_{0}}^{\infty} \frac{d r}{\beta_{0}(r) \cosh ^{2} r}=\infty
$$


for all $r_{0}>3$, and

$$
\left(\beta_{0} h^{2}\right)_{r r}^{\prime \prime}>\frac{\varepsilon}{\beta_{0} h^{2}}
$$

where $0<\varepsilon<1 / 4$ is small enough depending on the choice of $\beta_{0} \mid[0,5]$. We refer to [8] for a detailed construction of $\beta_{0}$ (denoted there by $\left.p_{0}\right)$; see also [19].

The smooth function $\ell:[0, \infty) \rightarrow[0, \infty)$ is constructed so that $\ell(r)=0$ for $r \in[0,3]$ and

$$
\ell^{\prime}=\frac{\varepsilon}{\beta_{0} h^{2}}
$$

on the interval $[5, \infty)$, with the same $\varepsilon$ as in (5.10). Finally, the two pieces are connected smoothly such that

$$
\ell^{\prime \prime} \geq \frac{-\ell^{\prime}\left(\beta_{0} h^{2}\right)_{r}^{\prime}}{\beta_{0} h^{2}} \quad \text { and } \quad 0 \leq \ell^{\prime} \leq \frac{\varepsilon}{\beta_{0} h^{2}}
$$

for all $r>0$. Then $\ell(r) \rightarrow \infty$ as $r \rightarrow \infty$ and $\beta(s, r)=\xi(s+\ell(r)) \beta_{0}(r)$ satisfies the conditions (5.6)-(5.9); see [8] for the details.

Next we complete the construction of $g$. Recall from (5.4) and (5.5) that

$$
\Omega=\{(s, r, \vartheta) \in M: r<3\} \cup\{s, r, \vartheta) \in M: s<-\ell(r)\}
$$

and hence $\beta \equiv 0$ and $g(s, r)=\frac{1}{2} \sinh (\sinh 2 r)$ in $\bar{\Omega}$ and $\beta>0$ in $M \backslash \bar{\Omega}$. Notice that integral curves of $W=R-\ell^{\prime} S$ starting at points in $\partial \Omega \cap\{(s, r, \vartheta) \in M: r>3\}$ will stay in $\partial \Omega$. Since

$$
\frac{1}{\beta h^{2}}-\ell^{\prime} \geq \frac{1}{\beta_{0} h^{2}}-\frac{\varepsilon}{\beta_{0} h^{2}}=\frac{1-\varepsilon}{\beta_{0} h^{2}}
$$

we conclude from (5.7) that all integral curves of $Z=\beta h^{2} R-S$ starting at points in $M \backslash \bar{\Omega}$ will enter $\Omega$ and stay in there, see p. 229 in [8]. As observed earlier, $\varrho$ and $g$ are constant along any integral curves of $Z$. This completes the construction of $g$ and the Riemannian metric of $M$. We refer to [19] for the proof of the curvature conditions (3.5) and (3.7).

We finish this section by collecting further properties of $g$ that will be used in Section 6. Recall from (5.1) and (5.3) that

$$
g(s, r)=\frac{1}{2} \sinh (\sinh 2 \varrho(s, r)),
$$

where $\varrho$ is a $C^{\infty}$-function, with $\varrho(s, r)=r$ for $0 \leq r \leq 3$, that satisfies the partial differential equation

$$
\varrho_{s}^{\prime}=\beta h^{2} \varrho_{r}^{\prime} .
$$

Hence we may apply the proof of Lemma 2.2 in [8] to the function $\varrho$. Since $\varrho_{r}^{\prime}(s, r) \equiv 1$ in $\Omega$, we get

$$
\begin{aligned}
\varrho_{r}^{\prime} & \geq 1, \quad \text { and } \\
\varrho(s, r) & \geq r
\end{aligned}
$$


in $M$. Furthermore,

$$
\begin{aligned}
& g_{r}^{\prime}=\varrho_{r}^{\prime} \cosh (2 \varrho) \cosh (\sinh 2 \varrho), \quad \text { and } \\
& \frac{g_{r}^{\prime}}{g}=2 \varrho_{r}^{\prime} \operatorname{coth}(\sinh 2 \varrho) \cosh 2 \varrho .
\end{aligned}
$$

\section{Construction of $Q$-subsolutions $\varphi_{a, c}$}

In this section we construct the functions $q_{a}:[0, \infty) \rightarrow \mathbb{R}, a \in \mathbb{R}$, so that the resulting functions $\varphi_{a, c}$ satisfy the conditions in Theorem 2.3. For each fixed $a \in \mathbb{R}$, we first define $q=q_{a}$ piecewise on intervals $\left[0, T_{0}\right],\left[T_{0}, T_{1}\right],\left[T_{1}, T_{2}\right],\left[T_{2}, T_{3}\right]$, and $\left[T_{3}, \infty\right)$, where $T_{0}, \ldots, T_{3}$ depend only on $a$ and $B_{0}$, and then finally smooth out $q$ in neighborhoods of $T_{i}, i=0,1,2,3$. We denote both the piecewisely constructed functions and the final smooth functions by the same symbol $q$.

Recall from (4.2) that

$$
f(s)=f^{(a, c)}(s)=c \max \{0, \tanh (\delta(s-a))\} .
$$

with $\delta=\frac{1}{2\left(1+2 B_{0}\right)}$. Furthermore, by (4.3) and (4.6), we have

$$
\varphi_{s}^{\prime}\left(s^{\prime}, r, \vartheta\right)=f^{\prime}(s)>0 \quad \text { and } \quad \varphi_{s s}^{\prime \prime}\left(s^{\prime}, r, \vartheta\right)=f^{\prime \prime}(s)<0
$$

for $s>a$, where

$$
s^{\prime}=s+\int_{0}^{r} q(t) d t
$$

Hence

$$
\frac{\varphi_{s s}^{\prime \prime}\left(1+h^{2} q^{2}\right)}{\varphi_{s}^{\prime} h}\left[1+2 \mathcal{B}\left(|\nabla \varphi|^{2}\right)|\nabla \varphi|^{2}\right] \geq-\frac{1+h^{2} q^{2}}{h}
$$

in $M_{a}$. We conclude from Lemma 4.1 that $Q[\varphi]>0$ in $M_{a}$ if

$$
\frac{g_{r}^{\prime} h(\beta-q)}{g}-h q_{r}^{\prime}-h_{r}^{\prime} q-\frac{1+h^{2} q^{2}}{h}-2 \bar{B}_{0} \frac{\left|h^{3} q_{r}^{\prime} q^{2}-h_{r}^{\prime} q\right|}{1+h^{2} q^{2}}>0
$$

in $M_{a}$, where $\bar{B}_{0}=\max \left(B_{0}, 1 / 2\right)$.

It is straightforward to check that integral curves of vector fields $R-\tanh r S$, $r>0$, are horizontal (Euclidean) lines, i.e., the $x^{3}$-coordinate remains constant along an integral curve. Hence we define $q(r)=q_{a}(r)=-\tanh r$ for $r \in\left[0, T_{0}\right]$, where $T_{0} \geq 1$ will be chosen later. Then the surfaces $S_{s}^{a}$ coincide with horizontal Euclidean planes $x^{3} \equiv e^{-s}$ near $L$. Consequently, the functions $\varphi_{a}$ are smooth in $M_{a}$. We notice that

$$
q_{r}^{\prime}=-\cosh ^{-2} r, \quad 1+h^{2} q^{2}=\cosh ^{2} r, \quad-h q_{r}^{\prime}-h_{r}^{\prime} q=\cosh r,
$$

and

$$
\frac{h^{3} q_{r}^{\prime} q^{2}-h_{r}^{\prime} q}{1+h^{2} q^{2}}=0
$$


Furthermore, since $\beta \geq 0$, we get from (5.15) and (6.1) that $Q[\varphi]>0$ in $M_{a} \cap$ $\left\{\left(s^{\prime}, r, \vartheta\right): 0<r<T_{0}\right\}$ because there

$$
\begin{aligned}
& \frac{g_{r}^{\prime} h(\beta-q)}{g}-h q_{r}^{\prime}-h_{r}^{\prime} q-\frac{1+h^{2} q^{2}}{h}-2 \bar{B}_{0} \frac{\left|h^{3} q_{r}^{\prime} q^{2}-h_{r}^{\prime} q\right|}{1+h^{2} q^{2}} \\
& \quad \geq \operatorname{coth}(\sinh 2 \varrho) \sinh (2 \varrho) \operatorname{coth}(2 \varrho) 2 \varrho \varrho_{r}^{\prime} \frac{\sinh r}{\varrho} \geq \varrho_{r}^{\prime} \frac{\sinh r}{\varrho}>0 .
\end{aligned}
$$

Since $\varrho=r$ and hence $\varrho_{r}^{\prime}=1$ for $0 \leq r \leq 3$, we have $\varrho_{r}^{\prime} \varrho^{-1} \sinh r \rightarrow 1$ as $r \rightarrow 0$.

For $r \in\left[T_{0}, T_{1}\right]$, we define

$$
q(r)=q_{a}(r)=\frac{-\cosh T_{0} \sinh r}{\cosh ^{2} r} .
$$

Then

$$
\begin{aligned}
& q_{r}^{\prime}(r)=\cosh T_{0}\left(\sinh ^{2} r-1\right) \cosh ^{-3} r, \quad 1+h^{2} q^{2}=1+\cosh ^{2} T_{0} \tanh ^{2} r, \\
& -h q_{r}^{\prime}-h_{r}^{\prime} q=\cosh T_{0} \cosh ^{-2} r,
\end{aligned}
$$

and

$$
\frac{\left|h^{3} q_{r}^{\prime} q^{2}-h_{r}^{\prime} q\right|}{1+h^{2} q^{2}}=\frac{\cosh ^{3} T_{0} \tanh ^{2} r\left(\tanh ^{2} r-\cosh ^{-2} r\right)+\cosh T_{0} \tanh ^{2} r}{1+\cosh ^{2} T_{0} \tanh ^{2} r} \leq \cosh T_{0} .
$$

Again since $\beta \geq 0$, we may estimate the left hand side of (6.1) from below to obtain

$$
\begin{aligned}
& \frac{g_{r}^{\prime} h(\beta-q)}{g}-h q_{r}^{\prime}-h_{r}^{\prime} q-\frac{1+h^{2} q^{2}}{h}-2 \bar{B}_{0} \frac{\left|h^{3} q_{r}^{\prime} q^{2}-h_{r}^{\prime} q\right|}{1+h^{2} q^{2}} \\
& \geq \cosh T_{0}\left(2 \varrho_{r}^{\prime} \operatorname{coth}(\sinh 2 \varrho) \cosh (2 \varrho) \tanh r+\cosh ^{-2} r\right. \\
& \left.\quad-\frac{1+\cosh ^{2} T_{0} \tanh ^{2} r}{\cosh T_{0} \cosh r}-2 \bar{B}_{0}\right) \\
& \quad>\cosh T_{0}\left(\cosh 2 r+\cosh ^{-2} r-\cosh ^{-2} T_{0}-\tanh ^{2} r-2 \bar{B}_{0}\right)>0
\end{aligned}
$$

in $M_{a} \cap\left\{\left(s^{\prime}, r, \vartheta\right): T_{0}<r<T_{1}\right\}$ provided $T_{0}=T_{0}\left(B_{0}\right) \geq 1$ is large enough.

For $r \in\left[T_{1}, T_{2}\right]$, we let $q=q_{a}$ be a $C^{\infty}$ continuation of $q \|\left[0, T_{1}\right]$ such that

$$
\frac{-\cosh T_{0} \sinh r}{\cosh ^{2} r} \leq q \leq 0
$$

and

$$
0<\left(\frac{-\cosh T_{0} \sinh r}{\cosh ^{2} r}\right)_{r}^{\prime}<q_{r}^{\prime}<\frac{\cosh T_{0}}{\cosh r}
$$

Thus

$$
\begin{aligned}
-h q_{r}^{\prime}-h_{r}^{\prime} & \geq-\cosh T_{0} \\
1 \leq 1+h^{2} q^{2} & \leq 1+\cosh ^{2} T_{0} \tanh ^{2} r \\
-\frac{1+h^{2} q^{2}}{h} & \geq-\frac{1+\cosh ^{2} T_{0} \tanh ^{2} r}{\cosh r},
\end{aligned}
$$


and

$$
\begin{aligned}
-2 \bar{B}_{0} \frac{\left|h^{3} q_{r}^{\prime} q^{2}-h_{r}^{\prime} q\right|}{1+h^{2} q^{2}} & =-2 \bar{B}_{0} \frac{h^{3} q_{r}^{\prime} q^{2}-h_{r}^{\prime} q}{1+h^{2} q^{2}} \geq-2 \bar{B}_{0}\left(h^{3} q_{r}^{\prime} q^{2}-h_{r}^{\prime} q\right) \\
& \geq-2 \bar{B}_{0} \cosh T_{0} \tanh ^{2} r\left(\cosh ^{2} T_{0}+1\right) .
\end{aligned}
$$

We choose $T_{1}=T_{1}\left(a, T_{0}\right)>T_{0}$ large enough so that $s^{\prime}+\ell(r) \geq 4$ for all $s^{\prime} \geq$ $a-\log \cosh T_{0}-1$ and $r \geq T_{1}$, which then implies that for all $s \geq a$ and $r \in\left[T_{1}, T_{2}\right]$ the point $\left(s^{\prime}, r, \vartheta\right)$ on any integral curve $\gamma_{a, s}$ of $X^{a}$, with

$$
s^{\prime}=s+\int_{0}^{r} q(t) d t \geq a-\log \cosh T_{0}-1,
$$

lies in the set where $\beta\left(s^{\prime}, r\right)=\beta_{0}(r)$. Furthermore, we also require that $T_{1}$ be so large that $\beta_{0}(r) \cosh ^{2} r \geq 1$ for all $r \geq T_{1}$. Then in $M_{a} \cap\left\{\left(s^{\prime}, r, \vartheta\right): T_{1}<r<T_{2}\right\}$, with $T_{1}$ large enough, we have

$$
\begin{aligned}
\frac{g_{r}^{\prime} h(\beta-q)}{g}-h q_{r}^{\prime}-h_{r}^{\prime} q-\frac{1+h^{2} q^{2}}{h}-2 \bar{B}_{0} \frac{\left|h^{3} q_{r}^{\prime} q^{2}-h_{r}^{\prime} q\right|}{1+h^{2} q^{2}} \\
\geq 2\left(\beta_{0}-q\right) \varrho_{r}^{\prime} \operatorname{coth}(\sinh 2 \varrho) \cosh (2 \varrho) \cosh r-\cosh T_{0} \\
\quad-\frac{1+\cosh ^{2} T_{0} \tanh ^{2} r}{\cosh r}-2 \bar{B}_{0} \cosh T_{0} \tanh ^{2} r\left(\cosh ^{2} T_{0}+1\right) \\
\geq 2 \cosh 2 r \cosh ^{-1} r-\cosh T_{0}-\frac{1+\cosh ^{2} T_{0} \tanh ^{2} r}{\cosh r} \\
-2 \bar{B}_{0} \cosh T_{0} \tanh ^{2} r\left(\cosh ^{2} T_{0}+1\right)>0
\end{aligned}
$$

Here we used estimates $\beta_{0}-q \geq \cosh ^{-2} r$ and $2 \varrho_{r}^{\prime} \operatorname{coth}(\sinh 2 \varrho) \cosh (2 \varrho) \geq 2 \cosh 2 r$ for $r \geq T_{1}$. The upper interval bound $T_{2}$ is determined by $q\left(T_{2}\right)=0$. Such $T_{2}$ exists since $q$ grows strictly faster than

$$
r \mapsto \frac{-\cosh T_{0} \sinh r}{\cosh ^{2} r}
$$

which tends to zero as $r \rightarrow \infty$. Since

$$
\int_{t}^{\infty} \beta_{0}(r) d r=\infty \text { and } \int_{t}^{\infty} \frac{d r}{\beta_{0}(r) \cosh ^{2} r}=\infty
$$

for every $t>3, \beta_{0}(r)-1 / \cosh r$ changes its sign infinitely often, in particular, there are arbitrary large values of $r$, with $\beta_{0}(r)-1 / \cosh r=0$. We let $T_{3}>T_{2}$ be a zero of $\beta_{0}-1 / \cosh$ specified later. For $r \in\left[T_{2}, T_{3}\right]$ we let $q(r)=0$. Then

$$
\begin{gathered}
\frac{g_{r}^{\prime} h(\beta-q)}{g}-h q_{r}^{\prime}-h_{r}^{\prime} q-\frac{1+h^{2} q^{2}}{h}-2 \bar{B}_{0} \frac{\left|h^{3} q_{r}^{\prime} q^{2}-h_{r}^{\prime} q\right|}{1+h^{2} q^{2}} \\
=\frac{g_{r}^{\prime} h \beta_{0}}{g}-\frac{1}{h} \geq \frac{2 \cosh 2 r-1}{\cosh r}>0
\end{gathered}
$$

in $M_{a} \cap\left\{\left(s^{\prime}, r, \vartheta\right): T_{2}<r<T_{3}\right\}$. 
For $r \geq T_{3}$ we define $q(r)=\beta_{0}(r)-1 / \cosh r$. Then

$$
\begin{aligned}
\beta-q & =\beta_{0}-q=1 / \cosh r \\
-h q_{r}^{\prime}-h_{r}^{\prime} q & =-\beta_{0}^{\prime}(r) \cosh r-\beta_{0}(r) \sinh r, \\
-\frac{1+h^{2} q^{2}}{h} & =-\beta_{0}^{2}(r) \cosh r+2 \beta_{0}(r)-2 \cosh ^{-1} r,
\end{aligned}
$$

and

$$
\begin{aligned}
-2 \bar{B}_{0} \frac{\left|h^{3} q_{r}^{\prime} q^{2}-h_{r}^{\prime} q\right|}{1+h^{2} q^{2}} & \geq-2 \bar{B}_{0} \frac{\left|h^{3} q_{r}^{\prime} q^{2}\right|+\left|h_{r}^{\prime} q\right|}{1+h^{2} q^{2}} \\
& \geq-2 \bar{B}_{0}\left(\left|\beta_{0}^{\prime}(r)\right| \cosh r+\beta_{0}(r) \sinh r+2 \tanh r\right) .
\end{aligned}
$$

Hence

$$
\begin{aligned}
\frac{g_{r}^{\prime} h(\beta-q)}{g} & -h q_{r}^{\prime}-h_{r}^{\prime} q-\frac{1+h^{2} q^{2}}{h}-2 \bar{B}_{0} \frac{\left|h^{3} q_{r}^{\prime} q^{2}-h_{r}^{\prime} q\right|}{1+h^{2} q^{2}} \\
\geq & 2 \cosh 2 r-\beta_{0}^{\prime}(r) \cosh r-\beta_{0}(r) \sinh r \\
& -\beta_{0}^{2}(r) \cosh r+2 \beta_{0}(r)-2 \cosh ^{-1} r \\
& -2 \bar{B}_{0}\left(\left|\beta_{0}^{\prime}(r)\right| \cosh r+\beta_{0}(r) \sinh r+2 \tanh r\right)>0
\end{aligned}
$$

in $M_{a} \cap\left\{\left(s^{\prime}, r, \vartheta\right): r>T_{3}\right\}$ if $T_{3}$ is large enough. Finally, since the estimates in (6.2)-(6.6) involve $q$ and $q_{r}^{\prime}$ but not higher order derivatives of $q$, it is clear that $q$ can be smoothen out in neighborhoods of $T_{i}$ such that (6.1) holds in $M_{a}$. Hence $\varphi_{a, c}$ is a positive $Q$-subsolution in $M_{a}$ and continuous in $M$, with $\varphi_{a, c}=0$ in $M \backslash M_{a}$. Next we use the divergence theorem to show that $\varphi_{a, c}$ is a $Q$-subsolution in whole $M$. To this end, let $\eta \in C_{0}^{\infty}(M)$ be an arbitrary non-negative test function and let $U \Subset M$ be an open set such that spt $\eta \subset U$ and that $\partial\left(M_{a} \cap U\right)$ is smooth. Since $\varphi_{a, c}=0$ in $M \backslash M_{a}, \eta=0$ in $M \backslash U$, and $\eta \operatorname{div} \mathcal{A}\left(\left|\nabla \varphi_{a, c}\right|^{2}\right) \nabla \varphi_{a, c} \geq 0$ pointwise in $M_{a} \cap U$, we obtain from the divergence theorem that

$$
\begin{aligned}
& \int_{M}\left\langle\mathcal{A}\left(\left|\nabla \varphi_{a, c}\right|^{2}\right) \nabla \varphi_{a, c}, \nabla \eta\right\rangle d m=\int_{M_{a} \cap U}\left\langle\mathcal{A}\left(\left|\nabla \varphi_{a, c}\right|^{2}\right) \nabla \varphi_{a, c}, \nabla \eta\right\rangle d m \\
& \quad=-\int_{M_{a} \cap U} \eta \operatorname{div} \mathcal{A}\left(\left|\nabla \varphi_{a, c}\right|^{2}\right) \nabla \varphi_{a, c} d m+\int_{\partial\left(M_{a} \cap U\right)}\left\langle\eta \mathcal{A}\left(\left|\nabla \varphi_{a, c}\right|^{2}\right) \nabla \varphi_{a, c}, \nu\right\rangle d \sigma \\
& \quad \leq \int_{\partial\left(M_{a} \cap U\right)}\left\langle\mathcal{A}\left(\left|\nabla \varphi_{a, c}\right|^{2}\right) \nabla \varphi_{a, c}, \eta \nu\right\rangle d \sigma,
\end{aligned}
$$

where $d \sigma$ is the (Riemannian) surface measure and $\nu$ is the unit outer normal vector field on $\partial\left(M_{a} \cap U\right)$. Furthermore,

$$
\eta \nu=-\frac{\eta \nabla \varphi_{a, c}}{\left|\nabla \varphi_{a, c}\right|}
$$

on $\partial\left(M_{a} \cap U\right)$, and therefore

$$
\int_{\partial\left(M_{a} \cap U\right)}\left\langle\mathcal{A}\left(\left|\nabla \varphi_{a, c}\right|^{2}\right) \nabla \varphi_{a, c}, \eta \nu\right\rangle d \sigma=-\int_{\partial\left(M_{a} \cap U\right)} \eta \mathcal{A}\left(\left|\nabla \varphi_{a, c}\right|^{2}\right)\left|\nabla \varphi_{a, c}\right| d \sigma \leq 0 .
$$


We conclude that $\varphi_{a, c}$ is a $Q$-subsolution in the whole $M$. Finally,

$$
\int_{0}^{\infty} q_{a}(t) d t=\infty
$$

for all $a \in \mathbb{R}$ since $q_{a}(t)=\beta_{0}(t)-1 / \cosh t$ for $t \geq T_{3}$,

$$
\int_{T_{3}}^{\infty} \beta_{0}(t) d t=\infty
$$

and

$$
\int_{T_{3}}^{\infty} \frac{d t}{\cosh t} \leq \int_{0}^{\infty} \frac{d t}{\cosh t}=\pi / 2
$$

Furthermore,

$$
\int_{0}^{r} q_{a}(t) d t \leq \int_{0}^{r} \beta_{0}(t) d t+\int_{0}^{r} \frac{d t}{\cosh t}=: b_{r}<\infty
$$

independently of $a \in \mathbb{R}$. Hence the family $\left\{\varphi_{a, c}\right\}$ satisfies conditions (a), (b'), and (c) in Theorem 2.3.

\section{Construction of $Q$-supersolutions $\psi_{a, c}$}

The construction of the family of continuous $Q$-supersolutions $\psi_{a, c}, a \in \mathbb{R}, c>0$, is similar to that in [8] and [19]. It is based on the following theorem from e.g. Theorem 4.3 in [10]:

Theorem 7.1. Let $N$ be an $n$-dimensional Cartan-Hadamard manifold with sectional curvatures $\leq-1$. Let $\Omega \subset N$ be a domain with $C^{\infty}{ }_{\text {-smooth boundary such }}$ that $\bar{\Omega}$ is convex. Then the distance function $\rho: N \backslash \bar{\Omega} \rightarrow(0, \infty)$,

$$
\rho(x)=\operatorname{dist}(x, \bar{\Omega}),
$$

is $C^{\infty}$ and

$$
\Delta \rho \geq(n-1) \tanh \rho
$$

in $N \backslash \bar{\Omega}$.

Suppose then that $\bar{\Omega} \subset N$ is a convex set and $\rho=\operatorname{dist}(\cdot, \bar{\Omega})$ is a distance function as in Theorem 7.1. Define a continuous function $v: N \rightarrow[0, c)$ by setting $v=0$ in $\bar{\Omega}$ and $v(x)=c \tanh (\delta \rho(x))$ for $x \in N \backslash \bar{\Omega}$, where $c>0$ and $\delta=\delta\left(B_{0}\right)$ is a positive constant depending only on the constant $B_{0}$ in (1.4). Then in $N \backslash \bar{\Omega}$ we have

$$
\nabla v=c \delta \cosh ^{-2}(\delta \rho) \nabla \rho \quad \text { and } \quad|\nabla v|=c \delta \cosh ^{-2}(\delta \rho) .
$$

To compute $Q[v]$, we first observe that

$$
\begin{aligned}
\operatorname{Hess} v\left(\frac{\nabla v}{|\nabla v|}, \frac{\nabla v}{|\nabla v|}\right) & =\operatorname{Hess} v(\nabla \rho, \nabla \rho)=\nabla \rho\langle\nabla v, \nabla \rho\rangle-(\nabla \nabla \rho \nabla \rho) v \\
& =\nabla \rho\left(c \delta \cosh ^{-2}(\delta \rho)\right)=\frac{-2 c \delta^{2} \tanh (\delta \rho)}{\cosh ^{2}(\delta \rho)}
\end{aligned}
$$


and that

$$
\Delta v=\operatorname{div}\left(c \delta \cosh ^{-2}(\delta \rho) \nabla \rho\right)=c \delta \cosh ^{-2}(\delta \rho)(\Delta \rho-2 \delta \tanh (\delta \rho)) .
$$

Hence by (1.4), (4.7), and (7.1) we have

$$
\begin{aligned}
Q[v] & =\operatorname{div} \mathcal{A}\left(|\nabla v|^{2}\right) \nabla v=\mathcal{A}\left(|\nabla v|^{2}\right)\left\{\Delta v+2 \mathcal{B}\left(|\nabla v|^{2}\right)|\nabla v|^{2} \operatorname{Hess} v\left(\frac{\nabla v}{\mid \nabla v}, \frac{\nabla v}{|\nabla v|}\right)\right\} \\
& \geq \frac{c \delta \mathcal{A}\left(|\nabla v|^{2}\right)}{\cosh ^{2}(\delta \rho)}\left((n-1) \tanh \rho-2 \delta\left(1+2 B_{0}\right) \tanh (\delta \rho)\right) .
\end{aligned}
$$

Choosing $\delta=\min \left(1, \frac{1}{2\left(1+2 B_{0}\right)}\right)$ yields

$$
\operatorname{div} \mathcal{A}\left(|\nabla v|^{2}\right) \nabla v \geq 0
$$

in $N \backslash \bar{\Omega}$. Hence the function $\psi=c-v$ is a continuous positive function in $N$, a $Q$-supersolution in $N \backslash \bar{\Omega}, \psi=c$ in $\bar{\Omega}$, and $\psi(x) \rightarrow 0$ as $\operatorname{dist}(x, \bar{\Omega}) \rightarrow \infty$. By a similar argument based on the divergence theorem as in the previous section, we conclude that $\psi$ is, in fact, a $Q$-supersolution in whole $N$.

Thus to construct the family $\left\{\psi_{a, c}\right\}, a \in \mathbb{R}, c>0$, it is enough to find appropriate convex subsets of $M$. This is done in [8] as follows. Denote by $\alpha_{a}$ any integral curve of $-\nabla_{\Theta} \Theta=g g_{r}^{\prime}(R+\beta S)$ starting at $L(a)$. Furthermore, denote by $P_{a}$ the surface obtained by rotating $\alpha_{a}$ around $L$ and let $V_{a}$ be the component of $M \backslash P_{a}$ containing points $L(s)$, with $s>a$. Observe that $P_{a}$ is also obtained by rotating integral curves of $R+\beta S$ starting at $L(a)$ around $L$. It is proven on p. 235 in [8] that $\bar{V}_{a}$ is convex for every $a \in \mathbb{R}$. Next we observe that, for each fixed $a \in \mathbb{R}$, the set $M_{a}=\left\{x \in M: \varphi_{a}(x)>0\right\}$ is contained in $\bar{V}_{a-b}$ for some $b=b\left(a, B_{0}\right)$. This is seen by comparing the (Fermi) $s$-coordinates of points $\left(s^{\prime \prime}, r, \vartheta\right)$ and $\left(s^{\prime}, r, \vartheta\right)$ on integral curves $\alpha_{a-b}$ and $\gamma_{a, s}, s \geq a$, respectively. More precisely, $s^{\prime} \geq s^{\prime \prime}$ for all such points $\left(s^{\prime \prime}, r, \vartheta\right)$ and $\left(s^{\prime}, r, \vartheta\right)$ if $b=b\left(a, B_{0}\right)$ is large enough since $\beta_{0}(r)-q_{a}(r)=1 / \cosh r$ for $r \geq T_{3}=T_{3}\left(a, B_{0}\right)$ and $\int_{0}^{\infty} 1 / \cosh r d r=\pi / 2<\infty$. Finally, for each $a \in \mathbb{R}$ and $c>0$, let $\psi_{a, c}=c-v_{a, c}$, where $v_{a, c}=c \tanh \left(\delta \rho_{a}\right)$, where $\rho_{a}=\operatorname{dist}\left(\cdot, \bar{V}_{a-b}\right)$ and $\delta=\frac{1}{2\left(1+2 B_{0}\right)}$. Then, by the discussion above, $\psi_{a, c}$ is a continuous positive $Q$-supersolution in $M, 0 \leq \varphi_{a, c} \leq \psi_{a, c} \leq c, \psi_{a, c}=c$ in $\bar{V}_{a-b}$, and $\lim _{y \rightarrow x} \psi_{a, c}(y)=0$ for all $y \in M(\infty) \backslash\left\{x_{0}\right\}$.

In conclusion, the families $\left\{\varphi_{a, c}\right\}$ and $\left\{\psi_{a, c}\right\}$ satisfy the conditions in Theorem 2.3, and thus Theorems 1.1, 2.1, 2.2, and 2.3 are proven.

\section{References}

[1] Ancona, A.: Negatively curved manifolds, elliptic operators, and the Martin boundary. Ann. of Math. (2) 125 (1987), no. 3, 495-536.

[2] Ancona, A.: Positive harmonic functions and hyperbolicity. In Potential theory surveys and problems (Prague, 1987), 1-23. Lecture Notes in Math. 1344, Springer, Berlin, 1988. 
[3] Ancona, A.: Théorie du potentiel sur les graphes et les variétés. In École d'été de Probabilités de Saint-Flour XVIII-1988, 1-112. Lecture Notes in Math. 1427, Springer, Berlin, 1990.

[4] Ancona, A.: Convexity at infinity and Brownian motion on manifolds with unbounded negative curvature. Rev. Mat. Iberoamericana 10 (1994), no. 1, 189-220.

[5] Anderson, M. T.: The Dirichlet problem at infinity for manifolds of negative curvature. J. Differential Geom. 18 (1983), no. 4, 701-721.

[6] Anderson, M. T. And Schoen, R.: Positive harmonic functions on complete manifolds of negative curvature. Ann. of Math. (2) 121 (1985), no. 3, 429-461.

[7] Arnaudon, M., Thalmaier, A. and Ulsamer, S.: Existence of non-trivial harmonic functions on Cartan-Hadamard manifolds of unbounded curvature. Math. Z. 263 (2009), no. 2, 369-409.

[8] BorbéLy, A.: The nonsolvability of the Dirichlet problem on negatively curved manifolds. Differential Geom. Appl. 8 (1998), no. 3, 217-237.

[9] Casteras, J.-B., Holopainen, I. and Ripoll, J.: On the asymptotic Dirichlet problem for the minimal hypersurface equation in a Hadamard manifold. Preprint, arXiv: 1311.5693, 2013.

[10] ChOI, H. I.: Asymptotic Dirichlet problems for harmonic functions on Riemannian manifolds. Trans. Amer. Math. Soc. 281 (1984), no. 2, 691-716.

[11] Collin, P. And Rosenberg, H.: Construction of harmonic diffeomorphisms and minimal graphs. Ann. of Math. (2) 172 (2010), no. 3, 1879-1906.

[12] Dajczer, M., Hinojosa, P. A. And de Lira, J. H.: Killing graphs with prescribed mean curvature. Calc. Var. Partial Differential Equations 33 (2008), no. 2, 231-248.

[13] Dajczer, M., Lira, J.H. and Ripoll, J.: An interior gradient estimate for the mean curvature equation of Killing graphs. To appear in J. Anal. Math. Preprint at ArXiv: 1206.2900, 2012.

[14] Do Espírito-Santo, N. And Ripoll, J.: Some existence results on the exterior Dirichlet problem for the minimal hypersurface equation. Ann. Inst. H. Poincaré Anal. Non Linéaire 28 (2011), no. 3, 385-393.

[15] Eberlein, P. and O’Neill, B.: Visibility manifolds. Pacific J. Math. 46 (1973), 45-109.

[16] Gálvez, J. A. And Rosenberg, H.: Minimal surfaces and harmonic diffeomorphisms from the complex plane onto certain Hadamard surfaces. Amer. J. Math. 132 (2010), no. 5, 1249-1273.

[17] Heinonen, J., Kilpeläinen, T. And Martio, O.: Nonlinear potential theory of degenerate elliptic equations. The Clarendon Press, Oxford University Press, New York, 1993.

[18] Holopainen, I.: Asymptotic Dirichlet problem for the $p$-Laplacian on CartanHadamard manifolds. Proc. Amer. Math. Soc. 130 (2002), no. 11, 3393-3400.

[19] Holopainen, I.: Nonsolvability of the asymptotic Dirichlet problem for the $p$-Laplacian on Cartan-Hadamard manifolds. To appear in Adv. Calc. Var.

[20] Holopainen, I. And VÄHÄkangas, A.: Asymptotic Dirichlet problem on negatively curved spaces. J. Anal. 15 (2007), 63-110.

[21] Hsu, E. P.: Brownian motion and Dirichlet problems at infinity. Ann. Probab. 31 (2003), no. 3, 1305-1319. 
[22] Jenkins, H. And Serrin, J.: Variational problems of minimal surface type. II. Boundary value problems for the minimal surface equation. Arch. Rational Mech. Anal. 21 (1966), 321-342.

[23] Meeks, W. H. And Rosenberg, H.: The theory of minimal surfaces in $M \times \mathbb{R}$. Comment. Math. Helv. 80 (2005), no. 4, 811-858.

[24] Nelli, B. And Rosenberg, H.: Minimal surfaces in $\mathbb{H}^{2} \times \mathbb{R}$. Bull. Braz. Math. Soc. (N.S.) 33 (2002), no. 2, 263-292.

[25] Ripoll, J. And Telichevesky, M.: Regularity at infinity of Hadamard manifolds with respect to some elliptic operators and applications to asymptotic Dirichlet problems. Trans. Amer. Math. Soc. 367 (2015), no. 3, 1523-1541.

[26] Rosenberg, H., Schulze, F. And Spruck, J.: The half-space property and entire positive minimal graphs in $M \times \mathbb{R}$. J. Differential Geom. 95 (2013), no. 2, 321-336.

[27] Sa Earp, R. And Toubiana, E.: An asymptotic theorem for minimal surfaces and existence results for minimal graphs in $\mathbb{H}^{2} \times \mathbb{R}$. Math. Ann. 342 (2008), no. 2, 309-331.

[28] Schoen, R. And Yau, S. T.: Lectures on harmonic maps. In Conference Proceedings and Lecture Notes in Geometry and Topology, II. International Press, Cambridge, MA, 1997.

[29] SpRuck, J.: Interior gradient estimates and existence theorems for constant mean curvature graphs in $M^{n} \times \mathbb{R}$. Pure Appl. Math. Q. 3 (2007), no. 3 (special issue in honor of Leon Simon, Part 2), 785-800.

[30] Sullivan, D.: The Dirichlet problem at infinity for a negatively curved manifold. J. Differential Geom. 18 (1983), no. 4, 723-732 (1984).

[31] V̈̈нÄKangas, A.: Dirichlet problem on unbounded domains and at infinity. Preprint 499, Department of Mathematics and Statistics, University of Helsinki, 2009.

Received December 21, 2013; revised June 14, 2014.

Ilkka Holopainen: Department of Mathematics and Statistics, P.O. Box 68, 00014 University of Helsinki, Finland.

E-mail: ilkka.holopainen@helsinki.fi

Jaime B. Ripoll: UfRGS, Instituto de Matemática, Av. Bento Goncalves 9500, 91540-000 Porto Alegre-RS, Brasil.

E-mail: jaime.ripoll@ufrgs.br

I. H. supported by the Academy of Finland, project 252293; J. R. supported by the CNPq (Brazil) project 302955/2011-9. 